\title{
Depletion of aquaporin 1 decreased ADAMTS-4 expression in human chondrocytes
}

\author{
MASAHIKO HANEDA, SHINYA HAYASHI, TOMOYUKI MATSUMOTO, \\ SHINGO HASHIMOTO, KOJI TAKAYAMA, NOBUAKI CHINZEI, SHINSUKE KIHARA, \\ KAZUHIRO TAKEUCHI, KOTARO NISHIDA and RYOSUKE KURODA
}

Department of Orthopaedic Surgery, Kobe University Graduate School of Medicine, Kobe, Hyōgo 650-0017, Japan

Received July 27, 2017; Accepted January 16, 2018

DOI: $10.3892 / \mathrm{mmr} .2018 .8545$

\begin{abstract}
Inflammation serves an important role in the progression of osteoarthritis (OA), and IL-1 $\beta$ may act as a catabolic factor on cartilage, reducing the synthesis of primary cartilage components type II collagen and aggrecan. Aquaporin 1 (AQP1) is a $28-\mathrm{kDa}$ water channel formed of six transmembrane domains on the cell membrane. AQP1 is highly expressed in the anus, gallbladder and liver, and is moderately expressed in the hippocampus, ependymal cells of the central nervous system and articular cartilage. It was hypothesized that AQP1 may be highly expressed in OA cartilage and that it may increase the expression of catabolic factors during inflammatory OA progression. Therefore, the present study evaluated AQP1 functions in human OA articular chondrocytes. Primary chondrocytes were isolated from human hip and knee cartilage tissues, cultured and transfected with AQP1-specific small interfering RNA with or without subsequent IL-1 $\beta$ treatment. In vitro explant culture from hip cartilages were also prepared. Reverse transcription-polymerase chain reaction (RT-PCR) was performed to assess the expression of $A Q P$ genes in human articular cartilage, AQP1 immunohistochemistry of the cartilages and explant culture, as well as RT-quantitative PCR, western blotting and immunocytochemistry/immunofluorescence of OA chondrocytes to evaluate the expression of AQP1, and catabolic and anabolic factors. RT-PCR results demonstrated that $A Q P 0,1,3,7,9$, and 11 were expressed in OA chondrocytes. Immunohistochemistry revealed that $A Q P 1$ was highly expressed in the superficial to middle zones of $\mathrm{OA}$ articular cartilages. Additionally, $A Q P 1$ mRNA was significantly higher in OA cartilage and IL-1 $\beta$ treatment significantly increased
\end{abstract}

Correspondence to: Dr Shinya Hayashi, Department of Orthopaedic Surgery, Kobe University Graduate School of Medicine, 7-5-1 Kusunoki-cho, Chuo-ku, Kobe, Hyōgo 650-0017, Japan E-mail: shayashi@med.kobe-u.ac.jp

Key words: aquaporin 1, osteoarthritis, ADAMTS-4, chondrocyte, articular cartilage
AQP1 expression in hip explant cartilage. Furthermore, AQP1 downregulation decreased a disintegrin and metalloprotease with thrombospondin motifs (ADAMTS)-4 expression in OA chondrocytes, though it did not affect other associated genes. Immunofluorescence showed that AQP1 and ADAMTS-4 were co-localized. These findings indicated that AQP1 depletion may decrease ADAMTS-4 expression in human OA chondrocytes. Therefore, regulating AQP1 expression may be a strategy to suppress catabolic factors during OA progression.

\section{Introduction}

Osteoarthritis (OA) is the most frequently diagnosed musculoskeletal disorder. It is a multifactorial and slowly progressing degenerative joint disease (1). The role of inflammation in OA progression has been previously reported (2-5). IL-1 $\beta$ is an important cytokine in OA progression by acting as a catabolic factor to reduce the synthesis of two primary cartilage components, the type II collagen (COL2A1) and aggrecan (ACAN) (6). Moreover, the level of IL-1 $\beta$ in the synovial fluid, synovial membrane, cartilage, and subchondral bone layer is elevated in patients with OA (7). Previous reports demonstrated that treatment with IL- $1 \beta$ decreases COL2A1 expression (6) and increases MMP-13 expression (8). Therefore, IL-1 $\beta$ plays a critical role during OA progression.

In mammals, the aquaporin (AQP) family consists of thirteen subtypes (AQP0 to 12) and these proteins are expressed in various tissues (9). AQP expression has been previously reported in several animal models. A previous study demonstrated the expression of $\mathrm{AQP} 1$ and $\mathrm{AQP} 3$ in normal equine articular chondrocytes (10). Another study showed that $A Q P 1$, $A Q P 3$, and $A Q P 6$ were expressed in rat mandibular condylar cartilage, although only $A Q P 3$ mRNA was highly upregulated in rat OA cartilage (11). Additionally, an increase in $A Q P 1$ expression in meniscus tissue was demonstrated in an experimentally-induced rat OA model (12).

AQP1 is a membrane protein found in the red blood cells (13). It is a $28-\mathrm{kDa}$ water channel formed by six transmembrane domains with $\mathrm{N}$ - and $\mathrm{C}$-termini at the inner cytosolic site of the cell membrane (14). AQP1 is highly expressed in the anus, gallbladder, and liver, and it is moderately expressed in the hippocampus and ependymal cells of the central nervous 
system (15). The presence of AQP1 was also confirmed in human endothelia and certain water-transporting epithelia, mammary epithelium, articular chondrocytes, synoviocytes, and synovial microvessels (9). In the central nervous system, the expression of AQP1 is restricted to the choroid plexus region of the brain under normal conditions (16), but it is expressed in the microvascular endothelia and reactive astrocytes of brain tumors where it is thought to play a role in the development of vasogenic edema (17). Recently, enhanced AQP1 expression was observed in several diseases (18-20). AQP1 expression is increased in patients with autoimmune and alcoholic pancreatitis (18), Alzheimer's disease (19), and rheumatoid and psoriatic arthritis (20).

Several studies have demonstrated the expression of AQP1 and AQP3 in human articular cartilage (15,20-23). Mobasheri et al (15) showed moderate AQP1 expression in chondrocytes residing in the deep zone of the articular cartilage, adjacent to the subchondral bone in normal human femoral head articular cartilage. They also reported AQP1 expression in the synovial microvessels and synoviocytes in normal joints, but the expression was upregulated in the inflamed synovium of patients with RA, suggesting that edema formation and synovial fluid accumulation in RA joints may be a consequence of elevated AQP1 expression in the synovium (20). However, functional analysis was not performed in these previous studies $(15,20-23)$. We hypothesized that AQP1 is highly expressed in OA cartilages, and AQP1 may increase the expression of catabolic factors. Thus, we evaluated AQP1 functions in human OA chondrocytes.

\section{Materials and methods}

Human cartilage samples. OA cartilage tissues were obtained from the cartilage of lateral femoral condyles of patients with end-stage varus-type OA during total knee arthroplasty surgery $(n=31)$. The diagnosis of OA was based on clinical, laboratory, and radiographic evaluations. These patients showed apparent macroscopic OA progression. As a control, normal chondrocytes (as determined macroscopically) were obtained from the hip cartilage of patients that underwent surgery for femoral neck fracture with no recorded tumor complication $(n=12)$. All primary cartilage samples were obtained in accordance with the World Medical Association Declaration of Helsinki of ethical principles for medical research involving human subjects. The present study was approved by the ethical review board of Kobe University Graduate School of Medicine (Kobe, Hyōgo, Japan) and all patients provided written informed consent. The average age of OA patients in this study was 76.4 years, and the average age of patients with femoral neck fracture was 85.1 years, but there was no significant difference in age between the two groups.

Chondrocyte isolation and culture. Chondrocytes were isolated from the cartilage tissues and cultured. Previously, we have used OA chondrocytes isolated from the knee joint (24-26). Briefly, tissue samples were minced and digested in Dulbecco's modified Eagle's medium (DMEM, cat. no. D5746; Sigma-Aldrich; Merck KGaA, Darmstadt, Germany) containing $0.2 \%$ collagenase D (Roche, Basel, Switzerland) at $37^{\circ} \mathrm{C}$ for $3 \mathrm{~h}$ under $5 \% \mathrm{CO}_{2}$ in a Petri dish.
Dissociated cells were cultured in DMEM supplemented with $10 \%$ fetal bovine serum (FBS) (BioWhittaker; Lonza Group, Basel, Switzerland), $50 \mathrm{U} / \mathrm{ml}$ penicillin, and $0.05 \mathrm{mg} / \mathrm{ml}$ streptomycin. After overnight culture, non-adherent cells were removed, and adherent cells were further incubated in fresh medium. Five to six days after incubation, chondrocytes reached confluence and were re-plated into 6-well plates at a density of $2.0 \times 10^{5}$ cells/well in DMEM. All experiments were conducted using first-passage cells. To confirm the chondrocyte properties, the expression of type II collagen was assessed by reverse transcription-polymerase chain reaction (RT-PCR). We confirmed that type II collagen was expressed at a higher level in normal human hip chondrocytes than in OA knee chondrocytes, while type $\mathrm{X}$ collagen was expressed at a higher level in OA knee chondrocytes than in normal hip chondrocytes (data not shown).

RNA isolation and RT-PCR. To evaluate the expression of different $A Q P$ genes, OA chondrocytes $(\mathrm{n}=4)$ were cultured in 6-well plates with DMEM for $12 \mathrm{~h}$, and RNA was extracted using the QIA shredder and RNeasy Mini kit (Qiagen, Hilden, Germany) according to the manufacturer's instructions. For RT-PCR, $1 \mu \mathrm{g}$ of total RNA was reverse transcribed to first-strand complementary DNA (cDNA) using $1.25 \mu \mathrm{M}$ oligo-dT primers in $40 \mu \mathrm{l}$ PCR buffer II containing $2.5 \mathrm{mM}$ $\mathrm{MgCl}_{2}, 0.5 \mathrm{mM}$ dNTP mix, 0.5 U RNase inhibitor, and $1.25 \mathrm{U}$ MuLV reverse transcriptase (PerkinElmer, Inc., Foster City, CA, USA) at $42^{\circ} \mathrm{C}$ for $60 \mathrm{~min}$. The cDNA amplification was performed under the following PCR conditions: $94^{\circ} \mathrm{C}$ for $5 \mathrm{~min}$; followed by 35 cycles of $94^{\circ} \mathrm{C}$ for $30 \mathrm{sec}, 55^{\circ} \mathrm{C}$ for $30 \mathrm{sec}$, and $72^{\circ} \mathrm{C}$ for $30 \mathrm{sec}$; and a final extension at $72^{\circ} \mathrm{C}$ for $2 \mathrm{~min}$ using AmpliTaq Gold DNA Polymerase (cat. no. N8080241; Thermo Fisher Scientific, Inc., Rockford, IL, USA) and specific primers (Table I). The amplicons, along with the TrackIt $50 \mathrm{bp}$ DNA ladder (cat. no. 10488-043; Thermo Fisher Scientific, Inc.), were resolved using $3 \%$ polyacrylamide gel electrophoresis, and the signals were visualized using the LAS-3000 mini system (FujiFilm, Tokyo, Japan).

$R T$-quantitative PCR (RT-qPCR). The relative mRNA levels of human $A Q P 1$, matrix metalloproteinase (MMP)-3, MMP-13, a disintegrin and metalloprotease with thrombospondin motifs (ADAMTS)-4, ADAMTS-5, COL2A1, and ACAN in OA chondrocytes $(\mathrm{n}=19)$ and normal hip chondrocytes $(\mathrm{n}=4)$ were analyzed by SYBR-Green real-time PCR using the ABI prism 7500 sequence-detection system (Applied Biosystems, Foster City, CA, USA). The expression of the genes of interest was normalized against that of the GAPDH housekeeping gene using the comparative quantification cycle $(\mathrm{Cq})$-value method. The difference between the mean $\mathrm{Cq}$ values of the gene of interest and those of the housekeeping gene was denoted as $\Delta \mathrm{Cq}$, and the difference between the $\Delta \mathrm{Cq}$ of unknown samples and that of the calibrated sample was denoted as $\Delta \Delta \mathrm{Cq}$. The relative value of gene expression was calculated as the $2^{-\Delta \Delta \mathrm{Cq}}$ (27). Sequences of the primers used for detection are listed in Table I.

Histological evaluation of cartilage degeneration. The femoral condyles $(n=5)$ and femoral head $(n=5)$ were fixed in $4 \%$ paraformaldehyde for $24 \mathrm{~h}$, dehydrated in graded 
Table I. Sequence of primers used in reverse transcription- and reverse transcription-quantitative polymerase chain reaction.

Primer sequence $\left(5^{\prime}-3^{\prime}\right)$

\begin{tabular}{|c|c|c|}
\hline Gene & Forward & Reverse \\
\hline$G A P D H$ & GTTCGACAGTCAGCCGCATC & GGAATTTGCATGGGTGGA \\
\hline$A Q P O$ & TGTACTGGGTAGGCCCAATC & CСССТСCACGTAAACTCAGA \\
\hline$A Q P 1$ & TGGACACCTCCTGGCTATTG & GGGCCAGGATGAAGTCGTAG \\
\hline$A Q P 2$ & CACCCCTGCTCTCTCCATA & GAAGACCCAGTGGTCATCAAAT \\
\hline$A Q P 3$ & GCTGTATTATGATGCAATCTGGC & TAAGGGAGGCTGTGCCTATG \\
\hline$A Q P 4$ & GAAGGCATGAGTGACAGACC & ATTCCGCTGTGACTGCTTTC \\
\hline$A Q P 5$ & GCCACCTTGTCGGAATCTAC & TAAAGCATGGCAGCCAGGAC \\
\hline$A Q P 6$ & CACCTCATTGGGATCCACTT & GTTGTAGATCAGTGAGGCCA \\
\hline$A Q P 7$ & ATCTCTGGAGCCCACATGAA & GAAGGAGCCCAGGAACTG \\
\hline$A Q P 8$ & GTGCCTGTCGGTCATTGAG & CAGGGTTGAAGTGTCCACC \\
\hline$A Q P 9$ & TCTCTGAGTTCTTGGGCACG & GGTTGATGTGACCACCAGAG \\
\hline$A Q P 10$ & GATAGCCATCTACGTGGGTG & CACAGAAAGCAGACAGCAAC \\
\hline$A Q P 11$ & TCCGAACCAAGCTTCGTATC & TAGCGAAAGTGCCAAAGCTG \\
\hline$A Q P 12$ & ACTTGTTCTTCTGGCCGTAG & CTTACTGGAGTACGTGCAGG \\
\hline$M M P-3$ & ATTCCATGGAGCCAGGCTTTC & CATTTGGGTCAAACTCCAACTGTG \\
\hline$M M P-13$ & TGCTGCATTCTCCTTCAGGA & ATGCATCCAGGGGTCCTGGC \\
\hline ADAMTS-4 & GGCTAAAGCGCTACCTGCTA & GAGTCACCACCAAGCTGACA \\
\hline ADAMTS-5 & TATGACAAGTGCGGACTATG & TTCAGGGCTAAATAGGCAGT \\
\hline COL2Al & CCCAGAGGTGACAAAGGAGA & CACCTTGGTCTCCAGAAGGA \\
\hline$A C A N$ & GGCACTAGTCAACCCTTTGG & CTGAACCCTGGTAACCCTGA \\
\hline
\end{tabular}

AQP, aquaporin; MMP, matrix metalloproteinase; ADAMTS, a disintegrin and metalloprotease with thrombospondin motifs; COL2A1, cartilage components type II collagen; ACAN, aggrecan.

alcohol solutions, decalcified with $14 \%$ EDTA for 7 days, and embedded in paraffin wax. Histological sections were obtained at $10-\mu \mathrm{m}$ intervals.

Immunohistochemistry. Deparaffinized sections were digested with proteinase (Dako, Glostrup, Denmark) for $10 \mathrm{~min}$ and treated with $3 \%$ hydrogen peroxide (Wako Pure Chemical, Ltd., Industries, Osaka, Japan) to block endogenous peroxidase activity. The sections were treated with a 1:50 dilution of anti-AQP1 antibody (Santa Cruz Biotechnology, Inc., Dallas, TX, USA) at $4^{\circ} \mathrm{C}$ overnight, and subsequently treated with peroxidase-labeled anti-mouse immunoglobulin (Histofine Simple Stain MAX PO; Nichirei Bioscience, Tokyo, Japan) at room temperature for $30 \mathrm{~min}$. The signal was developed as brown-reaction products using a peroxidase substrate 3,3'-diaminobenzidine (Histofine Simple Stain DAB solution; Nichirei Bioscience), and the sections were examined under a microscope. Hematoxylin stain was used as a counter stain. Numbers of AQP1-positive cells were counted in five areas of high-magnification fields at both the superficial and deep zones of the cartilage tissue by triple-blinded observers. The average percentage of AQP1-positive cells from the total cell count was calculated. Positive cells superior of the tidemark were included in the assessment.
Explant culture. Normal hip cartilages $(n=3)$ were harvested under sterile conditions and explant pieces were placed in a culture dish containing DMEM with or without IL-1 $\beta$. Forty-eight $\mathrm{h}$ after stimulation, the explants were fixed in $4 \%$ paraformaldehyde for $24 \mathrm{~h}$, decalcified with 14\% EDTA for 7 days, dehydrated in graded alcohol solutions, and embedded in paraffin wax. Histological sections were obtained at $10-\mu \mathrm{m}$ intervals.

$I L-1 \beta$ stimulation. Chondrocytes were cultured in 6-well plates with or without stimulation with $10 \mathrm{ng} / \mathrm{ml}$ recombinant human IL-1 $\beta / \mathrm{IL}-1 F 2$ (cat. no. 201-LB; R\&D Systems) for $12 \mathrm{~h}$.

Small interfering RNA (siRNA) transfection. OA chondrocytes $(n=7)$ were placed onto 6-well plates at a density of $2.0 \times 10^{5}$ cells/well in DMEM supplemented with $10 \%$ FBS and $100 \mathrm{U} / \mathrm{ml}$ of penicillin-streptomycin. After subculturing at $37^{\circ} \mathrm{C}$ for $24 \mathrm{~h}$ under $5 \% \mathrm{CO}_{2}$, the medium was replaced with fresh serum-free medium, and chondrocytes were transfected with $0.5 \mathrm{nM}$ of non-specific control siRNA (negative control no. 2 siRNA; cat. no. AM4613; Thermo Fisher Scientific, Inc.) or AQP1-specific siRNA (cat. no. 4390824, assay ID: s1515 or s1516) using the Lipofectamine RNAiMAX transfection reagent (cat. no. 13778150) (both from Invitrogen; Thermo 
Fisher Scientific, Inc., Waltham, MA, USA). Twelve hours after transfection, the cells were treated with IL-1 $\beta$.

Western blot analysis. OA chondrocytes $(\mathrm{n}=3)$ were lysed in a buffer containing $25 \mathrm{mM}$ Tris, $1 \%$ Nonidet P-40, $150 \mathrm{mM}$ $\mathrm{NaCl}, 1.5 \mathrm{mM}$ EDTA, and a protease/phosphatase inhibitor mix (Roche Diagnostics, Basel, Switzerland). The lysates were centrifuged to remove cellular debris. The supernatants were collected, mixed with $4 \mathrm{X}$ electrophoresis sample buffer, electrophoresed on a 7.5-15\% polyacrylamide gradient gel (Biocraft, Tokyo, Japan), and transferred onto a blotting membrane (GE Healthcare Life Sciences, Little Chalfont, UK). Membranes were incubated with antibodies against AQP1 or $\alpha$-tubulin (cat. no. T9026; Sigma-Aldrich). Horseradish peroxidase (HRP)-conjugated goat anti-mouse immunoglobulin $\mathrm{G}$ antibody ( $\mathrm{IgG} \mathrm{Ab}$ ) was used as a secondary antibody and proteins were visualized using the ECL plus reagent (GE Healthcare Life Sciences) in a Chemilumino Analyzer LAS-3000 mini (Fujifilm). Protein expression was determined by semi-quantification of digitally captured images using the NIH ImageJ software (http://imagej.nih.gov/ij/). Three different samples were analyzed.

Immunocytochemistry/immunofluorescence (ICC/IF). OA chondrocytes $(n=3)$ were placed onto a glass-based dish (Iwaki, Tokyo, Japan) at a density of $2.0 \times 10^{5}$ cells/well in DMEM. Chondrocytes were transfected with non-specific control (0.5 nM) (Qiagen) or AQP1-specific siRNA-1 $(0.5 \mathrm{nM})$ using the Lipofectamine RNAiMAX transfection reagent. Twelve hours after transfection, the cells were left untreated or stimulated with $10 \mathrm{ng} / \mathrm{ml}$ of recombinant human IL-1 $\beta /$ IL-1F2 (R\&D Systems) for $12 \mathrm{~h}$. After stimulation, the chondrocytes were fixed with $4 \%$ paraformaldehyde at room temperature for $30 \mathrm{~min}$ and permeabilized with $0.25 \%$ Triton X-100 (Nacalai Tesque, Kyoto, Japan) for $30 \mathrm{~min}$. Fixed chondrocytes were then incubated with human anti-AQP1 mouse monoclonal antibody (1:50 dilution; Santa Cruz Biotechnology, Inc.) and mouse anti-ADAMTS-4 rabbit polyclonal antibody (1:500 dilution; Thermo Fisher Scientific, Inc.) in Can Get Signal immunostain Solution A (Toyobo, Osaka, Japan) overnight at $4^{\circ} \mathrm{C}$. After the primary antibody incubation, the chondrocytes were incubated with goat anti-mouse immunoglobulin Alexa Fluor Plus 555 (1:200 dilution) and goat anti-rabbit immunoglobulin Alexa Fluor Plus 488 (1:200 dilution) (both from Thermo Fisher Scientific, Inc.) as the secondary antibodies for $60 \mathrm{~min}$ at room temperature. The nuclei were stained with DAPI (Nacalai Tesque), and images were viewed and captured using a BZ-X700 microscope (Keyence, Osaka, Japan). Numbers of AQP1-positive cells, ADAMTS-4-positive cells, and DAPI-stained nuclei were counted in four areas of high-magnification fields by triple-blinded observers. The average percentage of AQP1-positive cells and ADAMTS-4-positive cells from the total nuclei count was calculated.

Statistical analysis. The Mann-Whitney U test for comparisons between two groups and one-way analysis of variance with Tukey-Kramer's post hoc test were applied to analyze differences between time points or between culture
A

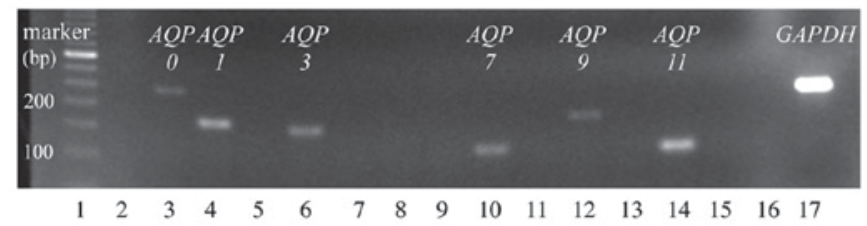

B

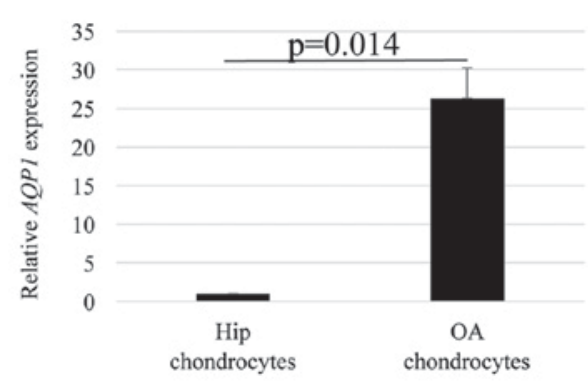

Figure 1. AQP expression in human articular cartilage. (A) The expression of various $A Q P$ genes in human articular cartilage. Lane 1 , size marker (50 bp ladder); lane 2, blank lane; lanes 3-15, PCR products obtained using the $A Q P$ $0-12$ primers; lane 16, blank lane; and lane 17, $P C R$ product obtained using the $G A P D H$ primer (n=4). (B) $A Q P 1$ mRNA expression in normal hip chondrocytes and $\mathrm{OA}$ chondrocytes as assessed by reverse transcription-quantitative $\mathrm{PCR}$. Data are presented as the mean \pm standard error. $\mathrm{P}=0.014$, as indicated. $\mathrm{AQP}$, aquaporin; $\mathrm{PCR}$, polymerase chain reaction.

conditions. P-values $<0.05$ indicated statistically significant differences. Results are presented as mean values \pm standard error (SE) with $95 \%$ confidence intervals (CI). Data analysis was performed using the Bell Curve for Excel software (Social Survey Research Information Co., Ltd., Tokyo, Japan).

\section{Results}

AQP1 was highly expressed in human OA chondrocytes. The expression of various $A Q P S$ was examined in OA chondrocytes from four patients with knee OA. Representative electrophoresis results with PCR products showed that $A Q P 0,1,3,7,9$ and 11 were expressed in OA chondrocytes (Fig. 1A). The average $A Q P 1$ mRNA expression in OA chondrocytes was significantly higher than that in normal chondrocytes from hip cartilage of four individuals ( $\mathrm{P}=0.014)$ (Fig. 1B). Immunohistochemistry results showed that surface layer of the articular cartilage was clearly degenerated in the OA group compared to that in the normal hip cartilage group. AQP1 was expressed in the superficial to middle layers in both OA $(n=5)$ and hip $(n=5)$ cartilage tissues (Fig. 2A), and the percentage of AQP1-positive cells was significantly higher in OA cartilages than in normal hip cartilages (AQP1-positive cell percentage: OA, 33.5\%; normal hip, 13.4\%; $\mathrm{P}=0.046$ ) (Fig. 2B). Furthermore, AQP1 was expressed in the superficial layers in hip explant cartilages with or without IL-1 $\beta$ treatment (Fig. $2 \mathrm{C}$ ). The percentage of AQP1-positive cells was significantly higher in explant cartilages treated with IL- $1 \beta$ than in those without IL-1 $\beta$ (AQP1-positive cell percentage: With IL-1 $\beta, 26.9 \%$; without IL-1 $\beta, 8.2 \%$; $P=0.0209$ ) (Fig. 2D). Together, these results indicated that AQP1 was highly expressed in human OA 
A

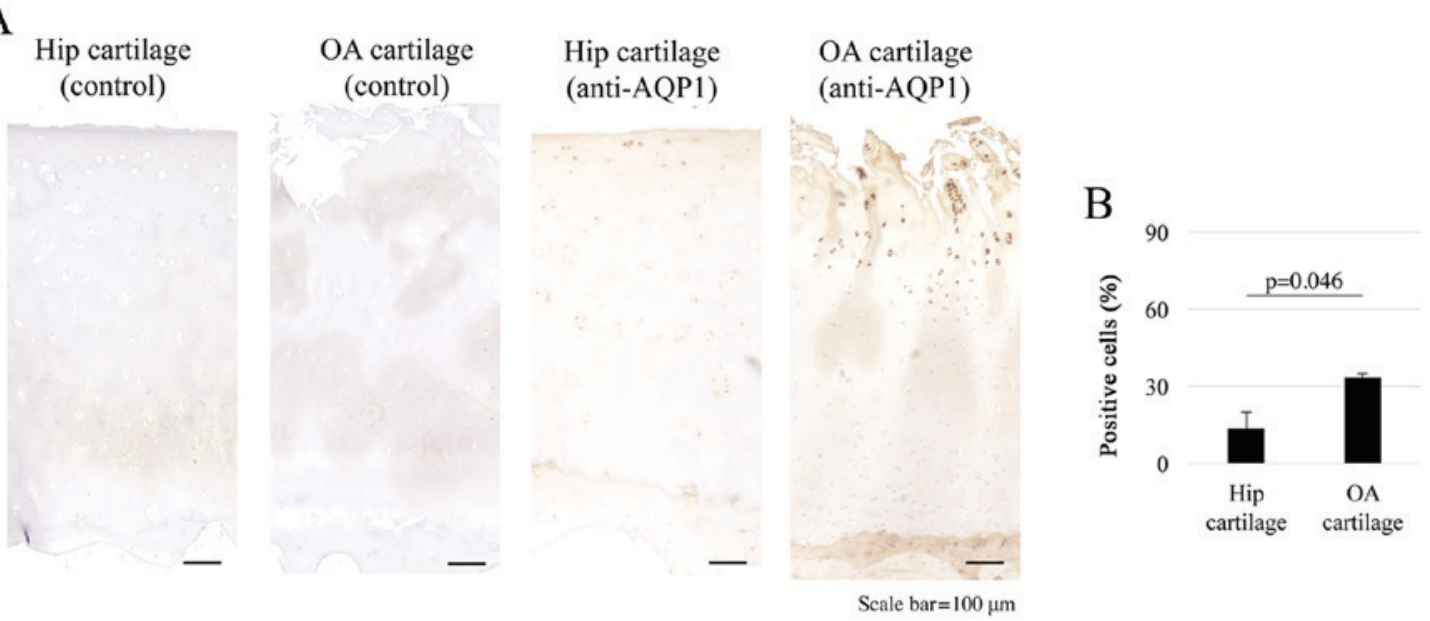

$\mathrm{C}$

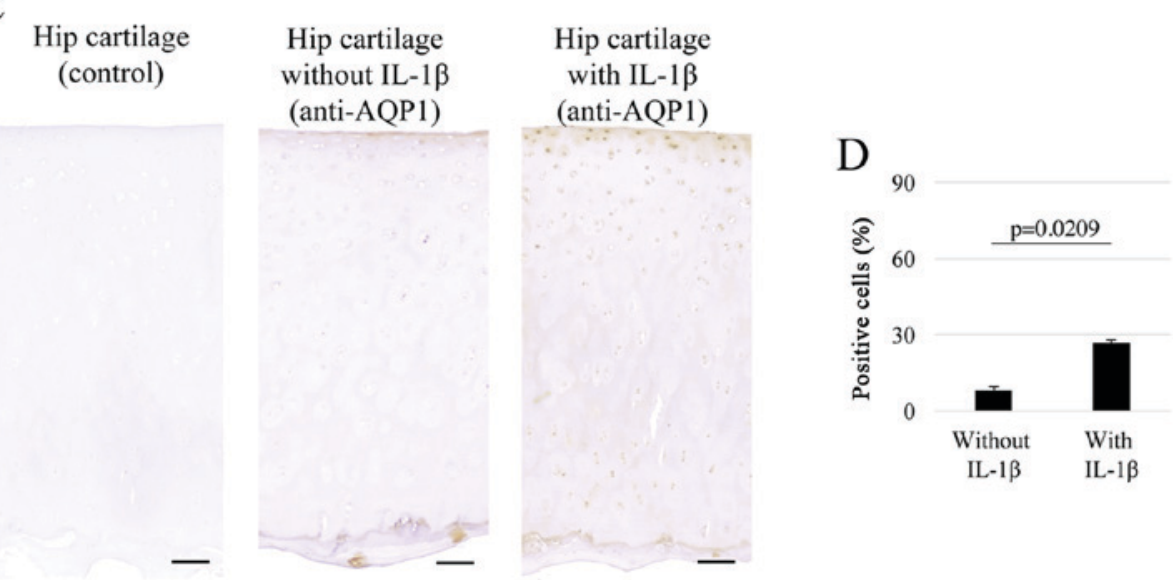

Figure 2. AQP1 immunohistochemistry of normal hip and OA cartilages. (A) Sections were unstained (secondary antibody only) or stained with anti-AQP1 antibody, and counterstained with hematoxylin and evaluated. Representative immunohistochemistry images and (B) the percentage of AQP1-positive cells in normal hip and OA articular cartilages (no. of positive cells/number of total cells with 95\% CI) are shown. (C) Representative AQP1 immunohistochemistry images of hip explant cartilages and (D) the percentage of AQP1-positive cells in the cartilages (relative to the total cell numbers with 95\% CI) are shown. Scale bars, $100 \mu \mathrm{m}$. AQP1, aquaporin 1; OA, osteoarthritis; CI, confidence interval; IL, interleukin.

chondrocytes, and the expression levels were increased in response to IL-1 $\beta$.

$I L-1 \beta$ increased the expression of $A Q P 1$ and catabolic factors in $O A$ chondrocytes. RT-qPCR analysis showed that the level of $A Q P 1$ mRNA was significantly higher in $\mathrm{OA}$ chondrocytes treated with $10 \mathrm{ng} / \mathrm{ml} \mathrm{IL-} 1 \beta$ than in untreated chondrocytes. At $4 \mathrm{~h}$ after IL-1 $\beta$ stimulation, the level of $A Q P 1 \mathrm{mRNA}$ was 5 -fold higher than that of the control $(0 \mathrm{~h}$; not treated with IL-1 $\beta$ ) (Fig. 3A). The mRNA levels of MMP-3, MMP-13, ADAMTS-4, and ADAMTS-5 were increased significantly following treatment with $10 \mathrm{ng} / \mathrm{ml}$ of IL-1 $\beta$ (Fig. 3B-E). The mRNA levels of $M M P-3$ and $M M P-13$ were increased in a time-dependent manner until $24 \mathrm{~h}$ after stimulation (Fig. 3B and C). The level of ADAMTS-4 mRNA was increased at $12 \mathrm{~h}$ after stimulation, but it was decreased at $24 \mathrm{~h}$ after stimulation (Fig. 3D). The level of ADAMTS-5 mRNA was increased at 12 and $24 \mathrm{~h}$ after stimulation (Fig. 3E). In contrast, the levels of COL2Al and ACAN mRNAs were significantly decreased at 12 and $24 \mathrm{~h}$ after stimulation (Fig. 3F and G). These results indicated that IL-1 $\beta$ stimulation increased the expression of $A Q P I$ and catabolic factors, but decreased the expression of anabolic factors in OA chondrocytes. It is important to note that although $A Q P 1$ mRNA expression peaked $4 \mathrm{~h}$ after IL-1 $\beta$ treatment, the level was still significantly higher than that of the control at $12 \mathrm{~h}$ after treatment. At $12 \mathrm{~h}$ post-IL-1 $\beta$ treatment, we also observed the peak of ADAMTS-4 and ADAMTS-5 mRNA expression, while that of COL2AI and ACAN decreased. Thus, we investigated changes in mRNA expression at $12 \mathrm{~h}$ after stimulation in subsequent experiments.

Downregulation of AQP1 decreased ADAMTS-4 expression in OA chondrocytes. To investigate the functions of AQP1 in chondrocytes, two AQP1-specific siRNAs were used to transfect the OA chondrocytes with or without IL- $1 \beta$ stimulation. IL-1 $\beta$ stimulation significantly increased the level of $A Q P I$ mRNA in OA chondrocytes (Fig. 4A). However, the level of $A Q P 1$ mRNA was significantly decreased in chondrocytes transfected with AQP1-specific siRNAs (Fig. 4A). The knockdown efficiency of $A Q P 1$ using AQP1 siRNA-1 (s1515) and -2 (s1516) with IL-1 $\beta$ stimulation was 94.3 and $88.5 \%$, respectively relative to the expression in the non-specific siRNA group with IL-1 $\beta$ stimulation (Fig. 4A). Western blot results 


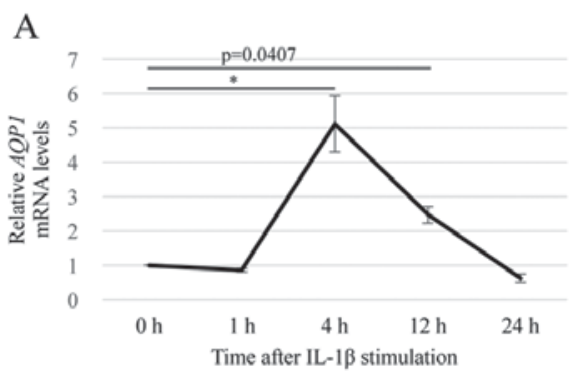

B

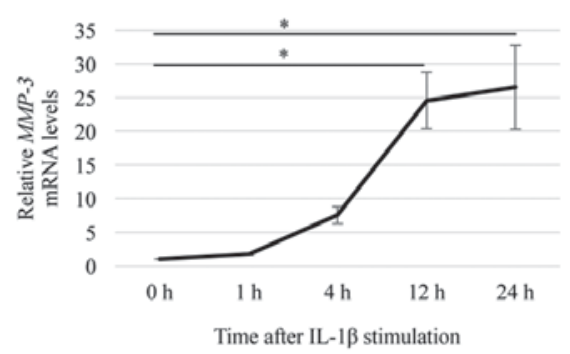

E

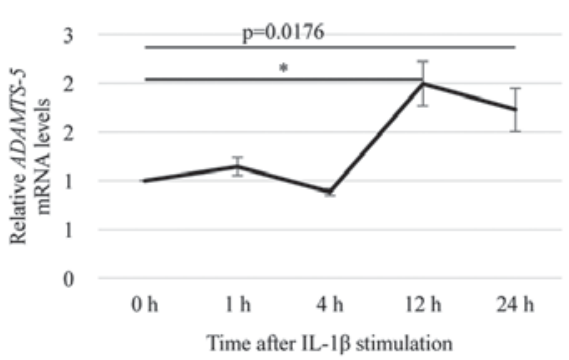

C

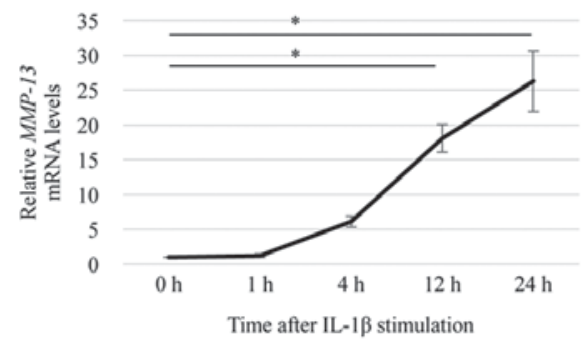

F

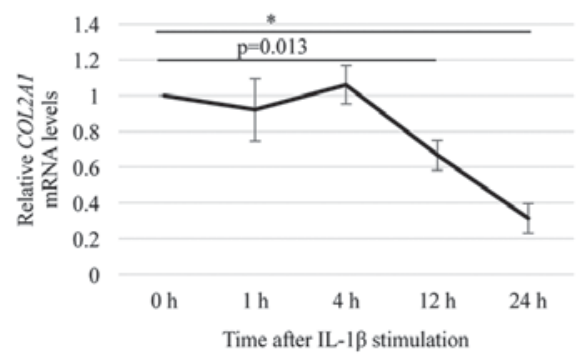

D

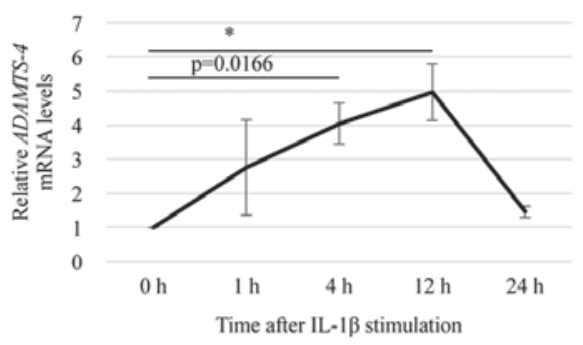

G

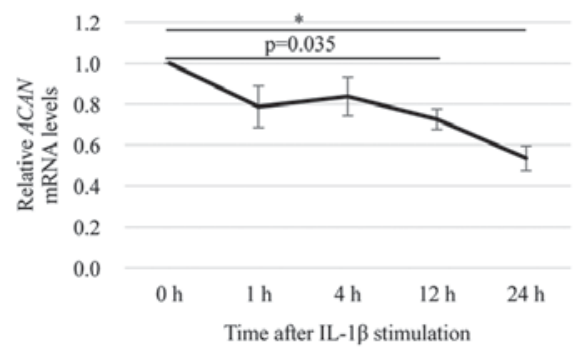

Figure 3. Effects of IL-1 $\beta$ stimulation on the expression of $A Q P 1$, catabolic factors and anabolic factors in OA chondrocytes. Changes in the mRNA level of (A) AQP1, (B) MMP-3, (C) MMP-13, (D) ADAMTS-4, (E) ADAMTS-5, (F) COL2A1 and (G) ACAN in OA chondrocytes stimulated with IL-1ß for 1, 4, 12 or $24 \mathrm{~h}$ were assessed by reverse transcription-quantitative polymerase chain reaction. GAPDH was used as the endogenous control. The value for each gene expression level without IL-1 $\beta$ stimulation $(0 \mathrm{~h})$ normalized to $G A P D H$ was set as 1 . Data are presented as the mean \pm standard error $(\mathrm{n}=8)$. *P<0.001, as indicated. IL, interleukin; AQP1, aquaporin 1; OA, osteoarthritis; MMP, matrix metalloproteinase; ADAMTS, a disintegrin and metalloprotease with thrombospondin motifs; COL2A1, cartilage components type II collagen; ACAN, aggrecan.

verified that AQP1 siRNA-1 and -2 significantly decreased AQP1 expression at the protein level in chondrocytes with or without IL-1 $\beta$ stimulation when compared to the level in cells transfected with non-specific siRNA (Fig. 4B and C). These results indicated that the AQP1-specific siRNAs downregulated AQP1 at both the mRNA and protein levels. The level of ADAMTS-4 mRNA was also significantly decreased to 64.2 and $65.8 \%$ using AQP1 siRNA-1 and -2, respectively under IL-1 $\beta$ stimulation (Fig. 4F). However, the mRNA levels of $M M P-3, M M P-13, A D A M T S-5, C O L 2 A 1$, and ACAN were not significantly changed by the AQP1-specific siRNA transfection (Fig. 4D and E, and G-I). These results indicated that AQP1 downregulation decreased ADAMTS-4 expression in OA chondrocytes, but did not affect the expression of other related genes.

AQP1 and ADAMTS-4 were co-localized in chondrocytes. To investigate the relationship between AQP1 and ADAMTS-4, we assessed AQP1 and ADAMTS-4 expression with or without IL-1 $\beta$ stimulation in human articular chondrocytes using immunofluorescence. Representative single-color images showing DAPI, AQP1, and ADAMTS-4 staining, as well as merged images are shown (Fig. 5A). The results showed that in the absence of IL- $1 \beta$ stimulation, AQP1 was expressed, while ADAMTS- 4 was not. Following IL- $1 \beta$ stimulation, the expression of both AQP1 and ADAMTS-4 was increased and the two molecules co-localized. However, AQP1 and ADAMTS-4 expression was decreased with AQP1-specific siRNA transfection. The results showed that AQP1-specific siRNA transfection reduced the percentage of AQP1 and ADAMTS- 4 co-localization and ADAMTS-4 expression in OA chondrocytes (Fig. 5B and C).

\section{Discussion}

Geyer et al (21) showed that the increase in AQP1 expression in damaged tissues was restricted to chondrocytes in the superficial areas, while non-lesional regions of human OA of the knee displayed lack of AQP1 expression. Hagiwara et al (23) suggested that AQP1 is expressed in the articular cartilage of the knee in human, and the expression is localized in chondrocytes in both intact and early degenerative cartilage 
A

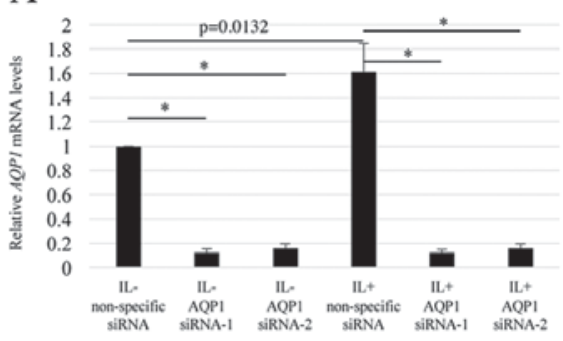

D

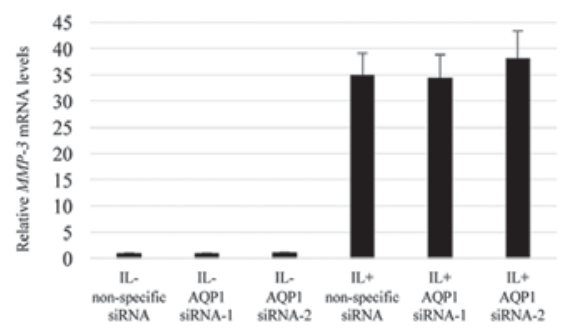

G

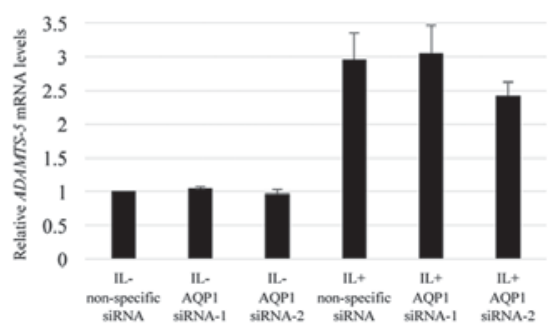

B

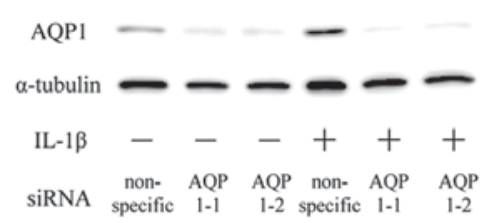

E

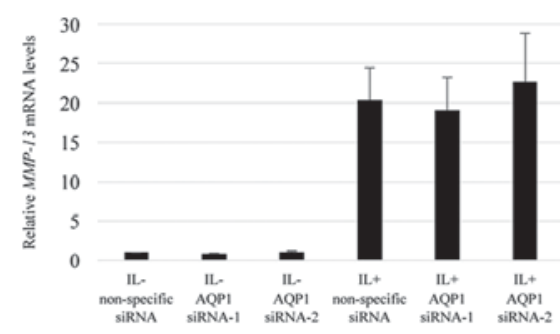

H

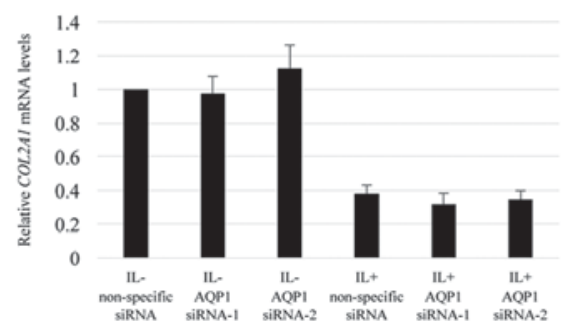

C

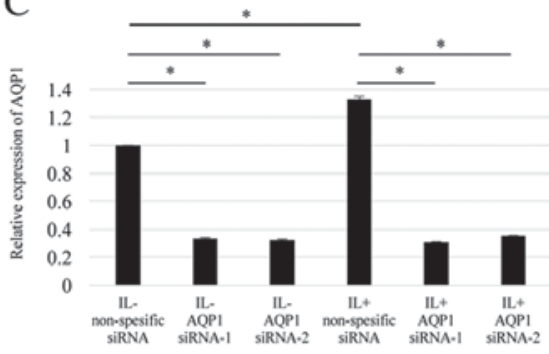

F

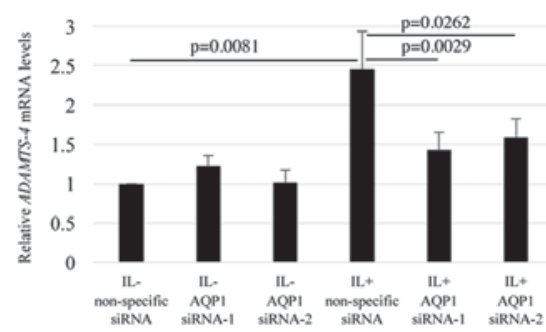

I

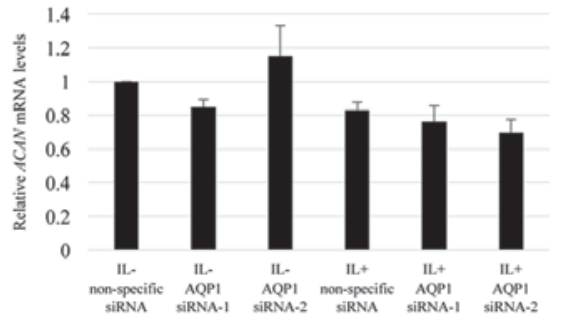

Figure 4. Effects of AQP1-specific siRNA transfection on IL-1ß-induced expression of catabolic factors in OA chondrocytes. OA chondrocytes were transfected with two different AQP1-specific siRNAs for $12 \mathrm{~h}$ and then stimulated without (IL-) or with IL-1 $\beta$ (IL+) for $12 \mathrm{~h}$. (A) AQPI knockdown efficiency was assessed by RT-qPCR. AQP1 protein expression levels were (B) assessed by western blotting and (C) the protein bands were semi-quantified (n=3). The AQP1 expression level in cells transfected with non-specific siRNA without IL-1 $\beta$ stimulation was set as 1 . Changes in the mRNA level of (D) $M M P$-3, (E) $M M P$-13, (F) ADAMTS-4, (G) ADAMTS-5, (H) COL2A1 and (I) ACAN were assessed by RT-qPCR. GAPDH was used as the endogenous control. The value for each gene expression level in cells treated with non-specific siRNA without IL-1 $\beta$ stimulation normalized to GAPDH was set as 1 . Data are presented as the mean \pm standard error $(\mathrm{n}=7)$. $\mathrm{P}<0.001$, as indicated. AQP1, aquaporin 1; siRNA, small interfering RNA; IL, interleukin; OA, osteoarthritis; RT-qPCR, reverse transcription-quantitative polymerase chain reaction; MMP, matrix metalloproteinase; ADAMTS, a disintegrin and metalloprotease with thrombospondin motifs; COL2A1, cartilage components type II collagen; ACAN, aggrecan.

regions. In this study, we demonstrated that $\mathrm{AQP1}$ was highly expressed in the superficial to middle zones of OA articular cartilages, and in the superficial zone of hip explant cartilages treated with IL-1 $\beta$. The regions where AQP1 is expressed may be related to progression of OA. We also observed that OA chondrocytes had significantly higher levels of $A Q P 1$ mRNA compared to those in normal chondrocytes from hip cartilage.

Recent reports have also described various AQP1 functions other than its role in water-dependent homeostasis (28-30). Meng et al (30) demonstrated that AQP1 enhances the migration of bone marrow mesenchymal stem cells by regulating the focal adhesion kinase and $\beta$-catenin. Another study found enhanced MMP-2 and -9 expression upon AQP1 downregulation in LTEP-A2 and LLC lung cancer cell lines (29). In the present study, we demonstrated that the AQP1 downregulation decreased ADAMTS-4 expression in OA chondrocytes, but did not affect the expression of other catabolic genes. Further, we observed that AQP1 and ADAMTS-4 co-localized and the expression of ADAMTS-4 was decreased upon AQP1 downregulation in OA chondrocytes. Based on these results, AQP1 may directly affect ADAMTS-4 expression in OA chondrocytes.

ACAN is the principal cartilage extracellular matrix proteoglycan that gives cartilage its characteristic compressibility, while ADAMTS is a family of proteases (31). A previous study showed that downregulation of ADAMTS-5 expression protects mice from arthritis-induced ACAN degradation (32). In vitro, downregulation of ADAMTS-4 and ADAMTS-5 was associated with ACAN cleavage prior to collagen degradation by MMP-13 $(33,34)$. ADAMTS-4 is predominantly expressed in OA cartilage, while ADAMTS-5 is constitutively expressed in both OA cartilage and normal hip cartilage. Further, the active form of ADAMTS-4 is overexpressed in OA chondrocytes, which directly correlates with the degradation of cartilage tissue (34). MMP and ADAMTS proteases cleave ACAN at different sites within the protein core (35). These findings supported our 
B

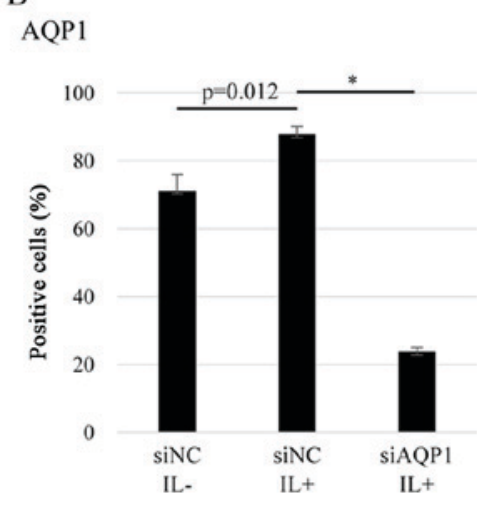

$\mathrm{C}$

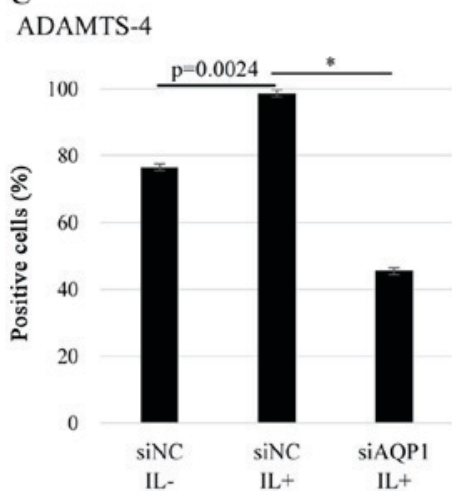

Figure 5. Immunostaining of OA human articular chondrocytes. DAPI, AQP1 and ADAMTS-4 staining are shown in blue, red and green, respectively. (A) OA articular chondrocytes were either transfected with non-specific control siRNA (siNC) without (IL-) or with IL-1 $\beta$ stimulation (IL+) for $12 \mathrm{~h}$, or transfected with siAQP1 with IL-1 $\beta$ stimulation (IL+) for $12 \mathrm{~h}$. The percentage of (B) AQP1- and (C) ADAMTS-4 positive cells (percentage of positive cells relative to DAPI-stained nuclei count with $95 \%$ confidence interval) is shown. Scale bar, $100 \mu \mathrm{m}$. Data are presented as the mean \pm standard error (n=3). "P<0.001, as indicated. AQP1, aquaporin 1; siRNA/NC, small interfering RNA/negative control; siAQP1, AQP1-specific siRNA; IL, interleukin; OA, osteoarthritis; ADAMTS-4, a disintegrin and metalloprotease with thrombospondin motifs 4; DAPI, 4',6-diamidino-2-phenylindole.

results that AQP1 knockdown suppressed IL-1 $\beta$-induced ADAMTS-4, but not ADAMTS-5, MMP-3, and MMP-13 expression.

Recently, Graziano et al (36) demonstrated a relationship between AQP1 and cartilage differentiation. However, we showed that AQP1 silencing did not affect $M M P-13$ expression in OA chondrocytes. Substantial phenotypic differences between differentiated chondrocytes used by Graziano et al (36) and OA chondrocytes in our study may explain the discrepancy. Cai et al (37) showed that AQP4 over-expression exacerbated the severity of adjuvant-induced arthritis in rat articular cartilages. This report supported our findings that AQP1 may control ADAMTS-4 expression in inflammatory chondrocytes. Nevertheless, further studies are needed to clarify the mechanism of IL-1 $\beta$-induced ADAMTS-4 regulation by AQP1.

Our study had several limitations. First, synovial fluid is produced in the synovium, and AQP is known to function in water metabolism in tissues. However, AQP1 functions in the synovial tissue were not assessed in the present study and should be addressed in a future study. Second, a previous report showed that AQP1 expression positively correlated with caspase-3 expression and activity, suggesting that AQP1 promoted caspase- 3 activation and thereby contributed to chondrocyte apoptosis and the development of OA (28). Thus,
AQP1 roles in chondrocyte apoptosis should also be evaluated in future studies.

In conclusion, we demonstrated that AQP1 was highly expressed in the superficial to middle zones of OA articular cartilages, and the level of $A Q P 1$ mRNA was significantly higher in $\mathrm{OA}$ than in normal chondrocytes from hip cartilages. Further, AQP1 downregulation decreased ADAMTS-4 expression in OA chondrocytes, but did not change the expression of other catabolic and anabolic genes evaluated. We also observed the co-localization of AQP1 and ADAMTS-4 expression, and a decrease in ADAMTS-4 expression upon AQP1 downregulation in OA chondrocytes. Our results indicated that AQP1 may play a role in maintaining the homeostasis of cartilage tissues; thus, catabolic factors may be suppressed by regulating AQP1 expression during OA progression.

\section{Acknowledgements}

The authors thank Mr. Takeshi Ueha, Ms. Kyoko Tanaka, Ms. Minako Nagata, and Ms. Maya Yasuda for their technical assistance, and Dr. Kazunari Ishida (Kobe Kaisei Hpspital) and Dr. Naoko Shima (Hyogo Prefectural Rehabilitation Central Hospital) for kindly providing the cartilage tissues. We would like to thank Editage (www.editage.jp) for English language editing. 


\section{References}

1. Altman R, Asch E, Bloch D, Bole G, Borenstein D, Brandt K Christy W, Cooke TD, Greenwald R, Hochberg M, et al: Development of criteria for the classification and reporting of osteoarthritis. Classification of osteoarthritis of the knee. Diagnostic and therapeutic criteria committee of the American Rheumatism association. Arthritis Rheum 29: 1039-1049, 1986

2. Berenbaum $\mathrm{F}$ and van den Berg WB: Inflammation in osteoarthritis: Changing views. Osteoarthritis Cartilage 23: 1823-1824, 2015.

3. Berenbaum F: Osteoarthritis as an inflammatory disease (osteoarthritis is not osteoarthrosis!). Osteoarthritis Cartilage 21: 16-21, 2013.

4. Goldring MB: The role of cytokines as inflammatory mediators in osteoarthritis: Lessons from animal models. Connect Tissue Res 40: 1-11, 1999.

5. Fernandes JC, Martel-Pelletier J and Pelletier JP: The role of cytokines in osteoarthritis pathophysiology. Biorheology 39: 237-246, 2002

6. Goldring MB, Birkhead J, Sandell LJ, Kimura T and Krane SM: Interleukin 1 suppresses expression of cartilage-specific types II and IX collagens and increases types I and III collagens in human chondrocytes. J Clin Invest 82: 2026-2037, 1988.

7. Wojdasiewicz P, Poniatowski ŁA and Szukiewicz D: The role of inflammatory and anti-inflammatory cytokines in the pathogenesis of osteoarthritis. Mediators Inflamm 2014: 561459, 2014.

8. Mengshol JA, Vincenti MP, Coon CI, Barchowsky A and Brinckerhoff CE: Interleukin-1 induction of collagenase 3 (matrix metalloproteinase 13) gene expression in chondrocytes requires $\mathrm{p} 38$, c-Jun $\mathrm{N}$-terminal kinase, and nuclear factor kappaB: Differential regulation of collagenase 1 and collagenase 3 . Arthritis Rheum 43: 801-811, 2000.

9. Agre P, King LS, Yasui M, Guggino WB, Ottersen OP, Fujiyoshi Y, Engel A and Nielsen S: Aquaporin water channels-from atomic structure to clinical medicine. J Physiol 542: 3-16, 2002.

10. Mobasheri A, Trujillo E, Bell S, Carter SD, Clegg PD, Martín-Vasallo P and Marples D: Aquaporin water channels $\mathrm{AQP} 1$ and AQP3, are expressed in equine articular chondrocytes. Vet J 168: 143-150, 2004.

11. Meng J, Ma X, Ma D and Xu C: Microarray analysis of differential gene expression in temporomandibular joint condylar cartilage after experimentally induced osteoarthritis. Osteoarthritis Cartilage 13: 1115-1125, 2005

12. Musumeci G, Leonardi R, Carnazza ML, Cardile V, Pichler K, Weinberg AM and Loreto C: Aquaporin 1 (AQP1) expression in experimentally induced osteoarthritic knee menisci: An in vivo and in vitro study. Tissue Cell 45: 145-152, 2013.

13. Preston GM, Carroll TP, Guggino WB and Agre P: Appearance of water channels in Xenopus oocytes expressing red cell CHIP28 protein. Science 256: 385-387, 1992.

14. Borgnia M, Nielsen S, Engel A and Agre P: Cellular and molecular biology of the aquaporin water channels. Annu Rev Biochem 68: 425-458, 1999.

15. Mobasheri A and Marples D: Expression of the AQP-1 water channel in normal human tissues: A semiquantitative study using tissue microarray technology. Am J Physiol Cell Physiol 286: C529-C537, 2004.

16. Oshio K, Watanabe H, Song Y, Verkman AS and Manley GT: Reduced cerebrospinal fluid production and intracranial pressure in mice lacking choroid plexus water channel Aquaporin-1. FASEB J 19: 76-78, 2005

17. Saadoun S, Papadopoulos MC, Davies DC, Bell BA and Krishna S: Increased aquaporin 1 water channel expression in human brain tumours. Br J Cancer 87: 621-623, 2002.

18. Ko SB, Mizuno N, Yatabe Y, Yoshikawa T, Ishiguro H, Yamamoto A, Azuma S, Naruse S, Yamao K, Muallem S and Goto $\mathrm{H}$ : Aquaporin 1 water channel is overexpressed in the plasma membranes of pancreatic ducts in patients with autoimmune pancreatitis. J Med Invest 56 (Suppl): S318-S321, 2009

19. Huysseune S, Kienlen-Campard P, Hébert S, Tasiaux B, Leroy K, Devuyst O, Brion JP, De Strooper B and Octave JN: Epigenetic control of aquaporin 1 expression by the amyloid precursor protein. FASEB J 23: 4158-4167, 2009.
20. Mobasheri A, Moskaluk CA, Marples D and Shakibaei M: Expression of aquaporin 1 (AQP1) in human synovitis. Ann Anat 192: 116-121, 2010

21. Geyer M, Grässel S, Straub RH, Schett G, Dinser R, Grifka J, Gay S, Neumann E and Müller-Ladner U: Differential transcriptome analysis of intraarticular lesional vs intact cartilage reveals new candidate genes in osteoarthritis pathophysiology. Osteoarthritis Cartilage 17: 328-335, 2009.

22. Trujillo E, González T, Marín R, Martín-Vasallo P, Marples D and Mobasheri A: Human articular chondrocytes, synoviocytes and synovial microvessels express aquaporin water channels; upregulation of AQP1 in rheumatoid arthritis. Histol Histopathol 19: 435-444, 2004

23. Hagiwara K, Shinozaki T, Matsuzaki T, Takata K and Takagishi K: Immunolocalization of water channel aquaporins in human knee articular cartilage with intact and early degenerative regions. Med Mol Morphol 46: 104-108, 2013.

24. Kawakita K, Nishiyama T, Fujishiro T, Hayashi S, Kanzaki N, Hashimoto S, Takebe K, Iwasa K, Sakata S, Nishida K, et al: Akt phosphorylation in human chondrocytes is regulated by $\mathrm{p} 53 \mathrm{R} 2$ in response to mechanical stress. Osteoarthritis Cartilage 20: 1603-1609, 2012.

25. Hayashi S, Fujishiro T, Hashimoto S, Kanzaki N, Chinzei N, Kihara S, Takayama K, Matsumoto T, Nishida K, Kurosaka M and Kuroda R: p21 deficiency is susceptible to osteoarthritis through STAT3 phosphorylation. Arthritis Res Ther 17: 314, 2015.

26. Iwasa K, Hayashi S, Fujishiro T, Kanzaki N, Hashimoto S, Sakata S, Chinzei N, Nishiyama T, Kuroda R and Kurosaka M: PTEN regulates matrix synthesis in adult human chondrocytes under oxidative stress. J Orthop Res 32: 231-237, 2014.

27. Livak KJ and Schmittgen TD: Analysis of relative gene expression data using real-time quantitative PCR and the 2(-Delta Delta C(T)) method. Methods 25: 402-408, 2001.

28. Gao H, Gui J, Wang L, Xu Y, Jiang Y, Xiong M and Cui Y: Aquaporin 1 contributes to chondrocyte apoptosis in a rat model of osteoarthritis. Int J Mol Med 38: 1752-1758, 2016.

29. Wei $X$ and Dong J: Aquaporin 1 promotes the proliferation and migration of lung cancer cell in vitro. Oncol Rep 34: 1440-1448, 2015.

30. Meng JH, Ma XC, Li ZM and Wu DC: Aquaporin-1 and aquaporin-3 expressions in the temporo-mandibular joint condylar cartilage after an experimentally induced osteoarthritis. Chin Med J (Engl) 120: 2191-2194, 2007.

31. Porter S, Clark IM, Kevorkian L and Edwards DR: The ADAMTS metalloproteinases. Biochem J 386: 15-27, 2005.

32. Glasson SS, Askew R, Sheppard B, Carito B, Blanchet T, Ma HL, Flannery CR, Peluso D, Kanki K, Yang Z, et al: Deletion of active ADAMTS5 prevents cartilage degradation in a murine model of osteoarthritis. Nature 434: 644-648, 2005.

33. Song RH, Tortorella MD, Malfait AM, Alston JT, Yang Z, Arner EC and Griggs DW: Aggrecan degradation in human articular cartilage explants is mediated by both ADAMTS- 4 and ADAMTS-5. Arthritis Rheum 56: 575-585, 2007.

34. Naito S, Shiomi T, Okada A, Kimura T, Chijiiwa M, Fujita Y, Yatabe T, Komiya K, Enomoto H, Fujikawa K and Okada Y: Expression of ADAMTS4 (aggrecanase-1) in human osteoarthritic cartilage. Pathol Int 57: 703-711, 2007.

35. Struglics A, Larsson S, Pratta MA, Kumar S, Lark MW and Lohmander LS: Human osteoarthritis synovial fluid and joint cartilage contain both aggrecanase- and matrix metalloproteinase-generated aggrecan fragments. Osteoarthritis Cartilage 14: 101-113, 2006.

36. Graziano ACE, Avola R, Pannuzzo G and Cardile V: Aquaporin1 and 3 modification as a result of chondrogenic differentiation of human mesenchymal stem cell. J Cell Physiol 233: 2279-2291, 2018.

37. Cai L, Lei C, Li R, Chen WN, Hu CM, Chen XY and Li CM: Overexpression of aquaporin 4 in articular chondrocytes exacerbates the severity of adjuvant-induced arthritis in rats: An in vivo and in vitro study. J Inflamm (Lond) 14: 6, 2017.

This work is licensed under a Creative Commons Attribution-NonCommercial-NoDerivatives 4.0 International (CC BY-NC-ND 4.0) License. 